Revue musicale OICRM

\title{
David Cronenberg et Howard Shore. Bref portrait d'une longue collaboration
}

\section{Solenn Hellégouarch}

Volume 2, numéro 2, 2015

Une relève

URI : https://id.erudit.org/iderudit/1060132ar

DOI : https://doi.org/10.7202/1060132ar

Aller au sommaire du numéro

\section{Éditeur(s)}

OICRM

ISSN

2368-7061 (numérique)

Découvrir la revue

Citer cet article

Hellégouarch, S. (2015). David Cronenberg et Howard Shore. Bref portrait d'une longue collaboration. Revue musicale OICRM, 2(2), 96-114.

https://doi.org/10.7202/1060132ar
Résumé de l'article

Après 45 ans de carrière, la filmographie de David Cronenberg compte 22 films, dont 15 ont été musicalisés par Howard Shore, qui a rejoint l'équipe du cinéaste en 1979. Si l'univers cronenbergien est aujourd'hui bien connu, l'apport de son compositeur demeure peu exploré. Or, la musique semble y jouer un rôle de toute première importance, le compositeur étant impliqué très tôt dans le processus cinématographique. Cette implication précoce est indicatrice du rôle central qu'occupent Shore et sa musique : comment le définir ? Plutôt que de recourir à une analyse des fonctions de la musique au cinéma, cet article explore les processus de création qui lui donnent naissance. Cronenberg et Shore, qui ont " tout appris en commun ", présentent ainsi des processus créateurs aux traits similaires, ou plus exactement des figures artistiques communes, ici exposées, les regroupant sous une seule vision artistique : l'autodidacte, l'expérimentateur, l'improvisateur, le peintre/sculpteur et l'artiste-artisan. 


\title{
David Cronenberg et Howard Shore. Bref portrait d'une longue collaboration
}

\author{
Solenn Hellégouarch
}

\begin{abstract}
Résumé
Après 45 ans de carrière, la filmographie de David Cronenberg compte 22 films, dont 15 ont été musicalisés par Howard Shore, qui a rejoint l'équipe du cinéaste en 1979. Si l'univers cronenbergien est aujourd'hui bien connu, l'apport de son compositeur demeure peu exploré. Or, la musique semble y jouer un rôle de toute première importance, le compositeur étant impliqué très tôt dans le processus cinématographique. Cette implication précoce est indicatrice du rôle central qu'occupent Shore et sa musique : comment le définir? Plutôt que de recourir à une analyse des fonctions de la musique au cinéma, cet article explore les processus de création qui lui donnent naissance. Cronenberg et Shore, qui ont «tout appris en commun", présentent ainsi des processus créateurs aux traits similaires, ou plus exactement des figures artistiques communes, ici exposées, les regroupant sous une seule vision artistique : l'autodidacte, l'expérimentateur, l'improvisateur, le peintre/sculpteur et l'artiste-artisan.
\end{abstract}

Mots clés : cinéma ; David Cronenberg ; musique de film ; processus créateur ; Howard Shore.

\begin{abstract}
In a career spanning 45 years, David Cronenberg's filmography includes 22 films. Since 1979, Howard Shore has scored 15 of them. Though Cronenberg's world is well known now, the weight of the contribution of his composer remains largely unmeasured. And this, despite the fact that music seems to play a primary role in the director's process since Shore is involved very early on. This unusually early involvement of the composer is indicative of the central role held by Shore and his music: how to define it? This paper does not seek to determine the functions of film music. Instead, it explores the creative processes that give rise to it. The creative processes of Cronenberg and Shore, who "learned everything together," thus have similar characteristics. More precisely, they present common artistic figures that gather them into a single artistic vision: the autodidact, the experimenter, the improviser, the painter/sculptor, and the artist-artisan.
\end{abstract}

Keywords: cinema; creative process; David Cronenberg; film music; Howard Shore. 
Le cinéaste David Cronenberg (1943-) réalise des films de fiction appartenant à un genre singulier qu'il a lui-même développé, celui de "l'horreur intérieure ». Depuis 1978, plusieurs collaborateurs, parfois regroupés sous le nom de Cronenberg crew (Théberge 2004, p. 131), participent à l'actualisation de sa vision insolite. Parmi ce groupe, un des premiers à consulter le script est le compositeur, Howard Shore (1946-), qui a rejoint l'univers cronenbergien en 1979¹. Depuis, le duo a été réuni sur 15 productions.

Bien que l'univers du réalisateur ait été maintes fois investigué, l'apport de Shore demeure peu examiné. Or, la musique semble y jouer un rôle de toute première importance, le compositeur étant impliqué très tôt dans le processus cinématographique. Cette implication précoce dans la création collective est indicatrice de la place et du rôle centraux qu'occupent Shore et sa musique. De la sorte, les partitions semblent ne pouvoir être considérées comme une simple illustration sonore des films, mais comme une composante tout à fait fondamentale, relançant dès lors la question du rôle de la musique au cinéma.

De nombreuses études s'attachent à définir un tel rôle. Mais bien souvent, les analystes étudient le rapport musique-image en termes de " fonctions »; chacun élabore ainsi sa théorie, son vocabulaire ${ }^{2}$ (Lissa 1967 ; Gorbman 1987 ; Chion [2003]2010), contribuant de la sorte à une définition fonctionnelle de la musique filmique et ce, tout en ignorant le processus créateur qui l'a façonnée. Mais comment, par quelle méthode passe-t-on de l'idée visuelle à l'idée musicale?

Le cadre de la relation continue entre réalisateur et compositeur permet de supposer la présence de constantes et d'une pensée musico-filmique consistante développées tout au long de la collaboration, surtout si la cohérence esthético-thématique du cinéaste, l'unité de leur œuvre, a déjà été questionnée ${ }^{3}$. Le portrait qui suit constitue une partie de nos recherches cherchant à déterminer un modèle du processus créateur de Cronenberg et Shore, ce qui nous a menée à constater la présence de constantes, de figures artistiques communes aux processus créateurs filmique et musical, reliant ainsi les deux créateurs en une seule vision artistique : l'autodidacte, l'expérimentateur, l'improvisateur, le peintre-sculpteur, l'artiste-artisan.

1 Les autres membres principaux de ce groupe sont Carol Spier, directrice artistique ; Peter Suschitzky, chef opérateur ; Ronald Sanders, monteur.

2 Nicholas Cook constate même un problème terminologique : " La littérature existante sur le multimédia souffre, à mon sens, de deux problèmes interreliés : l'appauvrissement terminologique incarné par la catégorisation traditionnelle de toutes les relations musique-image en termes de parallélisme ou de contrepoint dans le cadre de l'analyse filmique, et la présomption largement inconsciente (et certainement dépourvue d'esprit critique) selon laquelle de telles relations doivent être comprises en termes d'égémonie ou de hiérarchie plutôt qu'en termes d'interaction ", notre traduction de cet extrait : "The existing literature of multimedia suffers, as I see it, from two associated problems : the terminological impoverishment epitomized by film criticism's traditional categorization of all music-picture relationships as either parallel or contrapuntal, and a largely unconscious (and certainly uncritical) assumption that such relationships are to be understood in terms of hegemony or hierarchy rather than interaction" (Cook 1998, p. 107).

3 À ce propos, voir notamment Théberge 2004, p. 141-147. 
David Cronenberg fait carrière depuis maintenant 45 ans. De Stereo (1969) à Maps to the Stars (2014), sa filmographie compte 22 films auxquels s'ajoutent les courtsmétrages Transfer (1966) et From the Drain (1967). Tiraillé entre la littérature et les sciences à l'université (1963-1967), et à la suite de la découverte du film Winter Kept Us Warm (1965) de David Secter - une révélation (Rodley 1992, p. 10-11) - , il choisit finalement l'autodidactisme ${ }^{4}$ et le cinéma. Cronenberg se lance ainsi dans la réalisation filmique mais en ignore tout l'aspect technique. Il entreprend alors d'intégrer ce qu'il connaît déjà : la science et la littérature.

Ses principales influences sont littéraires, William Burroughs et Vladimir Nabokov : « Je me sentais vraiment soutenu et entraîné par leurs influences dans mon travail d'écriture, confie Cronenberg. C'est pourquoi les films ont représenté pour moi une libération » (Cronenberg cité dans Handling et Véronneau [1983]1990, p. 16). L'écriture permet ainsi de donner une première forme à ses idées. Mais une première difficulté surgit : "Comment faire une métaphore au cinéma? Je me rends compte que c'est par la création [...] d'images monstrueuses ». Le cinéaste précise sa pensée :

Les idées pures sont invisibles. Rien à filmer. C'est faisable en littérature, mais pas de la même façon au cinéma. [...] Je dois donner chair au verbe, puis filmer la chair, faute de filmer le verbe. [...] Je suis toujours à la recherche de la métaphore. Je dois la créer moi-même et c'est l'histoire qui la génère. [...] Je recherche la métaphore. Ce qui me conduit sans doute à une certaine monstruosité. C'est pour ça que j'invente ces choses (Cronenberg cité dans Labarthe 1999).

Ainsi, si l'écriture donne une première forme, concrète, à l'idée, la science en influence la matérialisation visuelle. Ce peut donc être des images monstrueuses - le choix du genre -, l'invention d'objets, créatures, maladies et mutations, ainsi que 1'utilisation, au cœur du récit, d'organisations et de personnages scientifiques douteux. Mais il reste un autre problème à surmonter : "C'était très naturel pour moi d'écrire un scénario, déclare Cronenberg. [...] La réalisation des films était la partie la plus difficile, celle de l'apprentissage ${ }^{5}$ " (Cronenberg cité dans Rodley 1992, p. 63).

La connaissance technique reste le seul obstacle à la matérialisation de la vision de Cronenberg, qui apprendra seul : il écrit le scénario, fait la prise de vues, assure le montage, enregistre le son. Avec Stereo, il passe du $16 \mathrm{~mm}$ au $35 \mathrm{~mm}$, format qui lui permet de rejoindre le milieu professionnel. Shivers (1975) le mène ensuite au cinéma commercial (avec une équipe de production, destiné à un plus large public), bien que ce film s'inscrive dans la lignée thématique des précédentes réalisations :

\footnotetext{
4 Cronenberg n'a pas suivi d'enseignement pratique et théorique du cinéma, il n'a pas été technicien, assistant-réalisateur/monteur, ni même critique de cinéma.

5 "It was very natural for me to write a script. [...] To realize the films was the hard part, the learning part "; notre traduction.
} 
la différence réside principalement dans l'évolution technique et le choix du genre 1'horreur. Avec Rabid (1976), ce premier long-métrage assoit les intérêts thématiques et esthétiques du cinéaste (dimensions scientifique, sexuelle, environnement aseptisé, révélateur d'un chaos à venir) qui participent à la définition du genre qu'il développe : celui de l'horreur corporelle, puis intérieure, né de la fusion des quatre genres que sont 1'horreur, la science-fiction, le fantastique, l'érotisme.

Cette fusion génère une image clinique et viscérale provoquant le malaise du spectateur confronté à son imaginaire refoulé. De fait, Cronenberg puise son inspiration dans l'être humain et son inconscient - l'horreur provient de l'intérieur -, alors matérialisé dans sa version la plus "réelle ", la plus crue. Si l'horreur graphique domine jusqu'à Videodrome (1982), les films suivants s'intéressent «aux contradictions de la psyché, aux conflits quasi freudiens qui font irruption au sein de l'individu " muant ainsi l'horreur viscérale en "horreur intérieure " (Handling et Véronneau [1983]1990, p. 8). Polymorphe, l'horreur traverse le corpus cronenbergien, directement liée à l'intérêt du cinéaste pour la biochimie et le corps humain qu'il conçoit comme le "premier fait de l'existence " (Labarthe 1999).

L'univers cronenbergien est un « univers mental », d'idées (Grünberg [1992]2002, p. 104). Mais sa traduction visuelle passe par le corps, la chair, substance palpable et réelle: "Pour moi, la réalité, c'est le corps [...], explique Cronenberg. Quelles que soient les pensées abstraites qui peuvent y être rattachées par la suite, je dois photographier les visages, les corps des gens. [...] La vraie violence, c'est la destruction du corps humain" (Cronenberg cité dans Ciment et Niogret 2007, p. 18). Et cette destruction peut prendre la forme d'une mutation menant irrémédiablement à la mort (The Dead Zone, The Fly), d'une terreur identitaire (Dead Ringers), d'une hallucination (Naked Lunch), d'un mensonge (M. Butterfly), d'un fantasme (Crash), d'un jeu vidéo immersif (eXistenZ), d'un esprit schizophrénique (Spider) ou névrotique ( $A$ Dangerous Method), de la violence criminelle ( $A$ History of Violence, Eastern Promises) ou capitaliste (Cosmopolis).

Le cinéma cronenbergien peut être décrit comme un laboratoire où chaque film est une expérience : expérience d'une vie, d'une mort, d'une croyance ou d'une philosophie que le cinéaste ne "partage pas nécessairement ", mais qu'il expérimente à travers ses personnages " pour connaître ses résultats " (Handling et Véronneau [1983]1990, p. 33-34). Cette expérimentation philosophique prend bien souvent la forme d'une exploration organique et mène Cronenberg à de nouvelles représentations du corps humain dont il expose la beauté intérieure; nous sommes ici au cœur du projet esthétique du cinéaste :

Pendant les 90 minutes d'un film, je veux qu[e] [les spectateurs] éprouvent d'abord une répulsion normale et qu'à la fin, ils voient la beauté ou une beauté possible dans ce qu'ils considéraient avant comme répugnant. C'est mon projet. C'est un projet esthétique. La transformation de l'esthétique humaine (Cronenberg cité dans Labarthe 1999).

Ce projet touche également la musique dans la mesure où Cronenberg et Shore cherchent à rompre avec la réalité musicale du spectateur - ce qu'il a l'habitude d'entendre. Il serait ainsi possible de parler d'un projet esthétique musico-visuel 
caractérisé, entre autres, par le refus de la redondance - en faveur de la créativité et de l'ambiguïté -, des conventions hollywoodiennes ${ }^{6}$ et de la musique préexistante ; nous y reviendrons. C'est aussi l'utilisation de textures, de sonorités et d'une instrumentation singulières - le mélange électroacoustique de Scanners (1981) et Crash (1996), le Synclavier de Videodrome, la juxtaposition orchestre-trio jazz de Naked Lunch (1992), le thérémine dans eXistenZ (1998) -, l'absence, bien souvent, de mélodie clairement identifiable, mémorisable et répétée. Il s'agit donc d'éviter les repères musicaux familiers afin d'en créer de nouveaux et de doter l'univers cronenbergien d'une musique qui n'a de lien qu'avec la réalité alternative créée par le réalisateur et cette esthétique nouvelle.

Avant 1979, et l'arrivée de Shore, Cronenberg se charge lui-même de la bande sonore de ses films. Avec Stereo, le choix coûteux du $35 \mathrm{~mm}$ le force à renoncer au son synchrone et à enregistrer une voix off - « ce sont des choses que vous n'osez plus faire dans le cinéma commercial. Mais à cette époque, je ne craignais pas de le faire ", note Cronenberg (cité dans Handling et Véronneau [1983]1990, p. 19). Crimes est conçu de la même manière mais bénéficie d'une trame sonore électronique dominée par des sons aquatiques. Shivers et Rabid sont dotés d'un patchwork de musique en stock (probablement issue de la compagnie Cinépix, qui produit ces deux films) qui, bien qu'en adéquation avec le contexte de ces films (musique plutôt atonale et dissonante), ne peut générer le même degré de subtilité qu'une musique originale, comme le note Paul Théberge : préformée, non destinée spécifiquement au film, son emplacement en regard du montage est parfois maladroit ; son caractère tend, en outre, à l'exagération (Théberge 2004, p. 142). Avec The Brood (1979), Cronenberg obtient un budget qui lui permet d'engager un compositeur.

\section{EXPÉRIMENTATION MUSICALE}

À ce moment précis, Shore n'a pas de formation particulière en musique filmique. Diplômé du Berklee College of Music (1969), où on enseigne autant la musique classique que le jazz, il a suivi le groupe rock Lighthouse en tournée (1969-1972) et travaillé pour le théâtre, la radio et la télévision - il est le directeur musical de Saturday Night Live de 1975 à 1980. Son premier essai filmique date de 1978 avec I Miss You, Hugs and Kisses (Murray Markowitz). Le second est donc The Brood.

Issu d'un milieu musical diversifié, Shore cherche un moyen d'exprimer librement ses idées, d'exploiter son potentiel créatif. C'est dans cette perspective qu'il se tourne vers Cronenberg et le cinéma :

C'est pourquoi Cronenberg était si parfait pour commencer - il n'avait jamais travaillé avec un compositeur et je n'avais jamais travaillé avec un réalisateur. Nous n'avions aucune idée préconçue. [...] Il n'y avait personne pour nous

6 À propos de la musique hollywoodienne voir, par exemple, Adorno et Eisler [1947]1972, Gorbman 1987, Kalinak 1992 ou encore Kassabian 2001. 
dire ce que nous pouvions faire ou non. C'était complètement expérimental (Shore cité dans Reynolds 2001) ${ }^{7}$.

Sa formation éclectique lui est d'une aide précieuse - lorsqu'il commence à œuvrer pour le cinéma, Shore est conscient des exigences du milieu. Mais pour la composition musico-filmique en tant que telle, il doit encore apprendre et, comme pour Cronenberg, il apprendra seul, ce qui explique en partie le caractère expérimental de ses premières œuvres filmiques.

C'est donc, ici aussi, l'inexpérience (absence de contraintes, d'idées préconçues) qui amène le compositeur à essayer de nouvelles pratiques musicales. Influencée par Herrmann, Bartók et Stravinsky, la partition de The Brood exploite des techniques compositionnelles avant-gardistes (dodécaphonisme, technique des cordes) qu'il ne pouvait utiliser ailleurs (Brown 1994, p. 337). Pour Scanners, des improvisations au synthétiseur sont enregistrées sur cassette, manipulées à l'aide d'un enregistreur multipiste, en faisant du montage sonore, et complétées par des parties orchestrales. Avec Videodrome, l'expérimentation atteint un nouveau degré avec une partition électronique réalisée au Synclavier. Le genre de ces films a pu décider, en partie, de la nature de leur partition, mais c'est surtout leur caractère expérimental - avec un budget limité - et l'absence de restrictions du réalisateur qui ont permis à Shore d'essayer diverses techniques.

Suite à cette première phase éminemment expérimentale, Shore s'essaye à d'autres univers sonores. Avec The Fly (1986), il passe à la composition pour orchestre et conçoit le film à la manière d'un opéra, en raison de son potentiel émotionnel et épique (Hellégouarch 2011a). Nous retrouvons une telle influence dans M. Butterfly (1993), qui combine les opéras italien et chinois avec la propre musique de Shore, et dans $A$ Dangerous Method (2011), où la présence de Wagner, justifiée narrativement c'est l'admiration commune de Carl Jung et Sabina Spielrein pour la Tétralogie -, est apportée par le compositeur par la citation, la transcription ou le mimétisme stylistique.

Dans ce film, Shore établit un lien biographique entre la genèse de l'opéra Siegfried et la relation amoureuse de Carl Jung et Sabina Spielrein. Pour cela, il utilise, entre autres, l'Idylle de Siegfried, composée par Wagner en 1869 pour célébrer l'anniversaire de son épouse et la naissance de leur premier enfant, Siegfried, puis intégrée dans l'acte III de Siegfried, lorsque le héros tombe amoureux de Brünnhilde - le motif symbolise 1'amour de cette dernière. Comme mythe et opéra, Siegfried est important dans les vies de Spielrein et Jung lorsqu'ils se rencontrent. Ainsi, dans le film, au fur et à mesure de ses occurrences, le motif de l'Idylle symbolise Siegfried, figure de l'amour (même impossible) partagé par les deux amants.

7 "That's why Cronenberg was so perfect to start with - he'd never worked with a composer, and I'd never worked with a director. We had no preconceptions. [...] There was nobody telling us what we could or couldn't do. It was completely experimental »; notre traduction. 


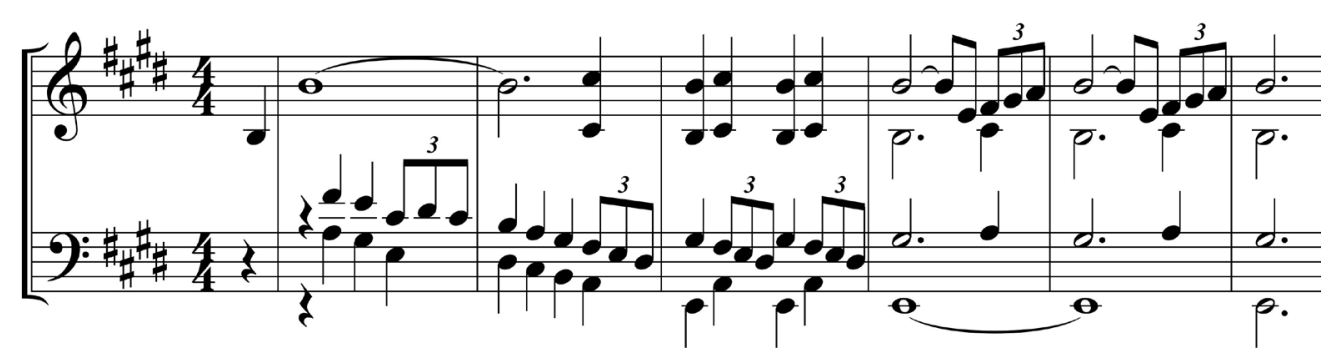

Figure 1: A Dangerous Method, thème de l'Idylle (mesures 1 à 6), piano-transcription.

S'il s'agit ici d'une des rares fois où la musique se réfère directement à celle d'un autre compositeur, ce n'est cependant pas la première fois que Shore fait appel à d'autres musiciens : Ornette Coleman dans Naked Lunch, le groupe Metric dans Cosmopolis. Ce dernier film marque un retour à l'électronique, délaissé depuis Crash: synthétiseurs, guitares électriques distortionnées et traitement électronique dominent cette bande sonore new wave rythmée, aux sonorités industrielles et urbaines. Dans Crash, Shore utilise une instrumentation singulière mêlant trois groupes constitués d'une harpe et de deux guitares électriques auxquels s'ajoutent le traitement électronique et le montage sonore. L'expérimentation sonore est ici aussi celle du langage, le compositeur exploitant des échelles modales renforçant la modernité et l'étrangeté du film.

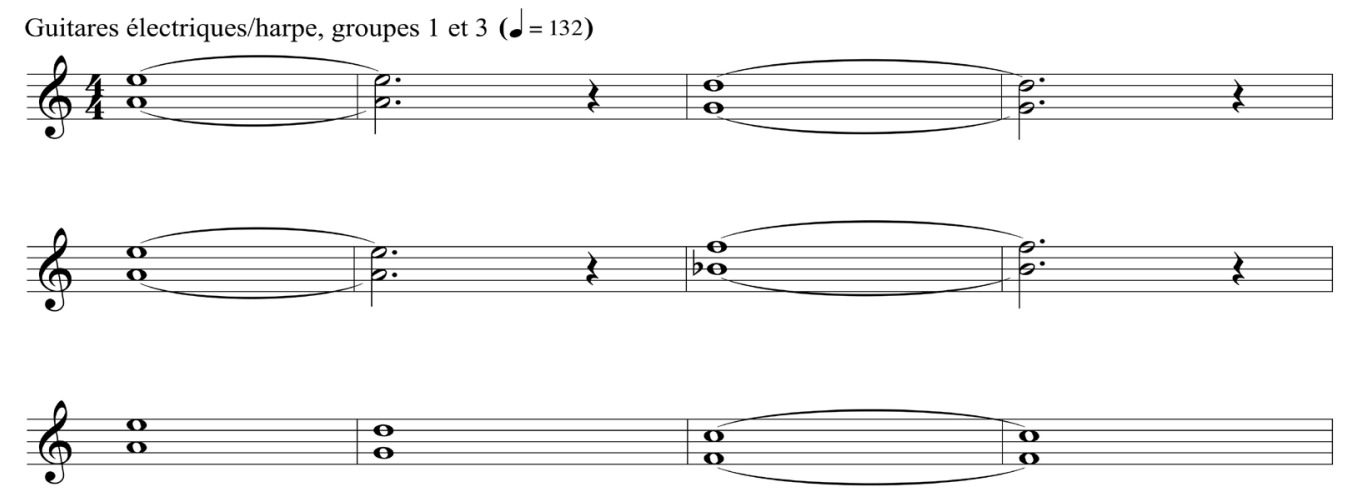

Figure 2: Thème de Crash (mesures 1 à 12)-transcription.

De la même manière, The Fly et Dead Ringers (1988) lui permettent d'explorer d'autres facettes du langage musical : la polytonalité, pour le premier, obtenue par renversement et superposition des triades (Schelle 1999, p. 328), et la création d'une "tonalité de l'effroi » (dread tonality), pour le second, générée par le monochromatisme des cordes et l'emploi de structures harmoniques créant une instabilité tonale : " travailler avec des quartes, des quintes, beaucoup de neuvièmes et de septièmes, beaucoup de secondes [...] en les faisant se chevaucher, en les jouant les unes contre les autres ${ }^{8} »($ Shore cité dans Schelle 1999, p. 348).

8 "[W]orking in fourths, fifths, a lot of ninths and sevenths, a lot of seconds [...] in a way of overlapping and playing them against each other »; notre traduction. 


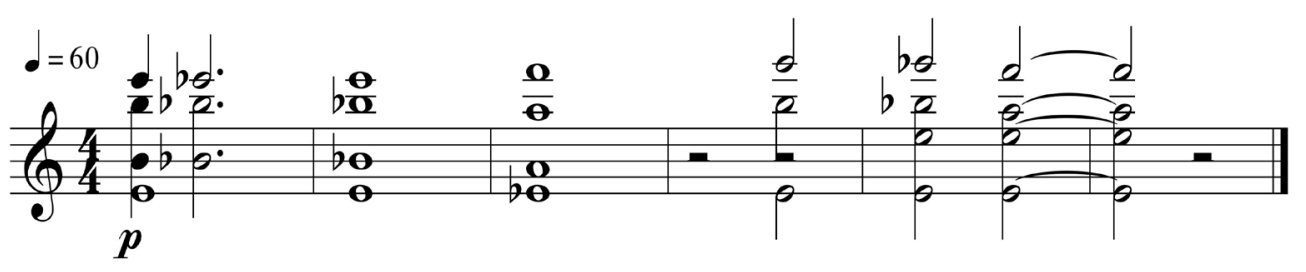

Figure 3 : Dead Ringers, cue 10 (01:02:38-01:03:01), cordes - transcription.

Dominée par les cordes, la musique de Dead Ringers contraste avec les musiques opératiques de The Fly et M. Butterfly par son austérité, réitérée dans Spider (2002), où elle est cependant obtenue par d'autres procédés - un ensemble instrumental restreint (quintette avec piano), le langage atonal.

Naked Lunch permet à Shore d'expérimenter une nouvelle approche sonore. Alors que le réalisateur adapte à l'écran la vie et l'œuvre de Burroughs, Shore collabore avec l'une de ses propres influences, Ornette Coleman. Le résultat de cette collaboration à quatre mains est une partition éclectique et chargée, une accumulation, un patchwork de diverses couches sonores reflétant l'ambiance hallucinatoire et la réalisation filmique inspirée du cut-up de Burroughs.

De film en film, Shore est un continuel apprenti: "Tout ce que je compose constitue un apprentissage. Je veux expérimenter, exprimer des idées. Ce n'est pas un état confortable. [...] C'est une incessante quête intérieure. " (Shore cité dans Willis-Sweete 2011). Cette expérimentation musicale est à relier avec le caractère polymorphe de l'horreur cronenbergienne et avec une volonté de dépassement qui unit les collaborateurs et encourage la reprise du travail en commun : "De continuer à travailler tous ensemble nous a poussé à donner le meilleur de nous-mêmes. [...] Avec David, chaque film vous pousse plus loin ${ }^{9}$ " (Shore cité dans Brown 1994, p. 338). Il n'y a donc aucun retour en arrière possible, il faut essayer autre chose. Ce dépassement est également généré par la liberté créatrice accordée par le cinéaste : « J'expérimente davantage dans les films de David parce qu'il me permet cette liberté et que ses films sont faits pour ces genres de sons ${ }^{10}$ " (Shore cité dans ibid., p. 328).

Pour Cronenberg, le dépassement implique un renouvellement. À la manière d'un thème et variations, ses films traitent des mêmes obsessions (ex. : le corps) à chaque fois soumises à un nouveau traitement, à divers corps, à différents récits. Selon le cinéaste, tous sont reliés à la musique de Shore qui connecte les grandes parties de l'œuvre globale : "Les gens perçoivent des liens à travers mes films et je vois tous ces liens connectés avec la musique de Howard. Je peux entendre les connexions ${ }^{11}$ " (Cronenberg cité dans Grünberg 2006, p. 182). De son côté, Shore souligne l'évolution

\footnotetext{
9 "Because we've all continued to work together, it drives everybody harder. [...] With David, in each movie you must go further»; notre traduction.

10 "I experiment more on David's movies because he allows me that freedom and his movies are suited to those kinds of sounds "; notre traduction.

11 "People see threads throughout my movies, and I see all those threads connected with Howard's score. I can hear the connections »; notre traduction.
} 
parallèle de sa musique et du travail du cinéaste : "Nous avons grandi ensemble en faisant des films, tout appris en commun. Vous verrez la même évolution dans son cinéma que dans ma musique " (Shore cité dans Joyard et Larcher 2000, p. 35). De l'un à l'autre, il n'est donc pas étonnant de trouver un processus créateur présentant des traits similaires : nous avons déjà exploré ceux de l'autodidactisme et de l'expérimentation. Ce dernier trait nous amène directement au suivant, l'improvisation.

\section{L'IMPROVISATION AU CEEUR DE LA COMPOSITION}

À l'instar du cinéaste qui incorpore les connaissances acquises ailleurs pour donner forme à son univers, Shore utilise ce qu'il connaît déjà pour s'arrimer à l'univers cinématographique, l'improvisation, procédé expressif et intuitif ${ }^{12}$ qu'il partage avec les jazzmen - notamment ses influences que sont Ornette Coleman, Gil Evans et Charles Mingus :

Lorsque les jazzmen improvisent [...] c'est une association libre de chaque musicien à l'autre, et la musique en devient très expressive. Donc, appliquez ça au cinéma et vous pouvez dire que la lumière passe de l'œil au cerveau, et j'exprime une idée intuitive, mon cerveau improvise ensuite sur ce que je vois à un moment particulier. Et puis je l'écris sur le papier et ça devient la composition (Shore cité dans Lauliac 2002, p. 94).

En outre, Shore perçoit un lien entrel'expérimentation, lejazz et l'idée du dépassement : "Venir du jazz et de l'improvisation signifie que vous essayez d'atteindre le prochain niveau. [...] Le jazz veut aller plus loin ${ }^{13}$ » (Shore cité dans Brown 1994, p. 341-341).

Premier lecteur des scénarios de Cronenberg, Shore n'a besoin de visionner les images qu'une fois. Il se concentre alors sur les éléments proprement visuels, qui lui dicteront ses choix musicaux :

Quand je regarde les séquences préliminaires d'un film, j'entends la musique. [...] La cinématographie, la façon dont les acteurs se déplacent, la façon dont ils disent leurs lignes, l'écriture, le montage - tout cela me dit immédiatement ce que doit être la musique (Shore cité dans Reynolds 2001) ${ }^{14}$.

Si Cronenberg est particulièrement attentif au corps - ce que nous retrouverons dans son rapport à la sculpture -, Shore est quant à lui sensible à la plastique du film - il est très graphique dans sa composition, comme nous le verrons. Pour Shore, la musique est un art visuel, elle « se trouve dans ce que l'on voit » (Willis-Sweete 2011) : ce sont

12 Le recours à l'intuition ne veut pas dire que le compositeur travaille ex nihilo. Bien au contraire, Shore, dont il faut ici se rappeler la formation éclectique, développe une écriture musicale faite d'emprunts à tous les styles, genres et époques de la musique, tel qu'il apparaît, en partie, dans notre brève description de ses partitions cronenbergiennes.

13 "Coming from a jazz background and to come through from improvisation means that you're trying to go to another level [...] jazz wants to go further "; notre traduction.

14 "When I watch the preliminary footage of a film, I hear the music. [...] The cinematography, the way the actors move, the way they say their lines, the writing, the editing - all of that tells me immediately what the music needs to $b e »$; notre traduction. 
les images qui provoquent une musique. Lors du visionnement, celle-ci est donc déjà présente, en puissance, et le compositeur n'a plus qu'à la révéler.

La première phase de composition est celle du "rêve", pendant laquelle Shore improvise sur un souvenir ${ }^{15}$ de l'image, se basant uniquement sur des sensations: "Pendant deux semaines, j'aurais consigné des heures d'improvisations et idées sur cassette, séquenceur, ou papier qui se rapportent au film de manière subliminale, onirique $^{16}$ " (Shore cité dans Small 1999, p. 264). Ce rapport distancé (sans image) et intuitif (l'improvisation) avec le film relève également d'un choix : "Si vous écrivez trop près de la scène, vous enlevez une partie de son pouvoir. Vous devez écrire sans être trop attentif. [...] Vous avez besoin d'une connexion avec le public qui regarde cette scène à un niveau beaucoup plus sublimina ${ }^{17}$ " (ibid.). L'improvisation, procédé instinctif, automatique et inconscient, permet de conserver cet effet subliminal et de maintenir un certain degré d'ambiguité.

Chez Cronenberg, cette recherche d'ambiguïé se traduit notamment par une réticence face à l'utilisation de musique préexistante, rattachée à la réalité personnelle des spectateurs (Grünberg 2006, p. 98). À l'opposé, une musique originale permet de contrôler davantage la réponse du spectateur. Contrairement à Hollywood, pour le cinéaste, cela signifie qu'il « doit éviter de dire au public ce qu'il doit ressentir » :

La musique doit apporter autre chose à la scène et au film de sorte que si elle n'était pas là la scène serait tout à fait différente. Si nous ne pouvons pas faire cela alors nous préférons ne pas avoir de musique. Voilà le niveau d'abstraction que nous cherchons. C'est ce que nous voulons vraiment la musique d'apporter au film (Cronenberg cité dans ibid., p. 100-101) ${ }^{18}$.

La musique ne doit donc pas être le miroir de l'action ; elle doit ajouter un autre niveau de signification. Shore partage cette même idée : "[La musique] exprime d'autres sentiments que ce qui est proposé visuellement par le metteur en scène. Je pense, qu'en faisant cela, vous élargissez la portée et les éléments émotionnels de l'histoire » (Aufort et Dupont 2011) ${ }^{19}$. L'improvisation traduit les idées, les sensations du film en sensations musicales et n'exclut aucune possibilité - Shore compose beaucoup de musique en avance dans un processus automatique et inconscient. Cette traduction

15 Parlant d'un concerto pour violoncelle, Shore explicite le même processus : "[Il] s'inspire de jardins que j'ai vus et que j'ai aimés. Ce n'est pas tant une visualisation qu'une émotion, [...] une concrétisation intérieure de la raison d'être du morceau. C'est une vision très intérieure. Un souvenir en fait » (Willis-Sweete 2011).

16 "Over a period of two weeks, I would have logged in hours of improvisations and ideas on tape, sequencer, or paper that related to the movie in a subliminal, dreamy way"; notre traduction.

17 "If you write too closely to the scene, you take away some of its power. You have to write to it without being too observant of it. [...] you need a connection to the audience who is watching it on a much more subliminal level "; notre traduction.

18 "The music must bring something else to the scene and to the movie, so that if it were not there the scene would be quite different. If we can't do that then we'd rather not have music. So that's how abstract we get. That's what we really want music to bring to the film "; notre traduction.

19 Sur cette question, voir notre propre analyse de Crash, Hellégouarch 2012. 
musicale de l'image-souvenir se fait l'expression des idées du film mais n'illustre pas une idée déjà donnée dans un rapport de redondance : la traduction musicale participe à l'édification de l'idée créatrice par un travail instinctif puis concret de la matière musicale. Il ne s'agit donc pas d'une traduction directe, littérale de l'image, mais de la transposition d'une idée d'un art à l'autre. L'idée en image devient idée en musique ${ }^{20}$.

Lorsque Shore improvise, il ne pense pas en termes de conventions ni de structure, mais en termes de sensations. C'est après cette étape qu'il entre dans une phase technique ; il analyse alors le résultat du premier jet créateur et choisit parmi l'ensemble des possibilités. À ce stade, ce qui compte c'est la visualisation musicale, les notes et les liens qui les unit sur le papier, le matériau - lorsqu'il compose, Shore pense à sa musique sous sa forme la plus épurée, soit en termes de contrepoint, harmonie, tempo, rythme, etc. (Temmerman 2003, p. 94). Ainsi, la musique est visuelle non seulement parce qu'elle naît de l'image, mais aussi parce qu'elle existe sur le papier : «Les gens pensent que la musique est ce que vous entendez [...]. Mais la musique est en fait une relation de valeurs sur une page ${ }^{21} »$ (Shore cité dans Reynolds 2001).

PEINDRE LA MUSIQUE

Dans l'instant du visionnement, Shore voit et retient les images, fixe des figures, des couleurs, des mouvements, des sensations. La visualisation externe se transforme ainsi en souvenir et rejaillit dans le geste spontané de l'improvisation. Le compositeur visualise alors son propre imaginaire, stimulé par l'image, et crée sa propre nécessité créatrice intérieure - il y a donc incitation à la création et non à l'imitation. Dans cet imaginaire ne subsistent que les sensations de l'expérience filmique. Expérimenter, c'est donc également faire l'expérience du film et la transmettre. Transposant ainsi l'univers cronenbergien dans son propre imaginaire intérieur, Shore se libère des limites imposées par le modèle filmique et se crée un autre modèle, sensuel. Nous ne sommes ainsi plus dans le champ de la figuration, mais dans celui de l'abstraction, où l'image est libérée de la matière : elle ne fait plus que résonner, une résonance que Shore ressent telle une vibration sonore.

C'est en termes de visualisation que Shore compare son travail à celui du peintre, rapprochement que l'on pressentait à propos de la musique définie telle des « relations de valeurs sur une page ». Dans l'atelier du peintre-compositeur, différents pinceaux permettent d'extérioriser ses images intérieures. Les plus fins sont utilisés pour la musique de concert, où l'on « entre littéralement avec une infime précision dans tous les contrepoints » : destinée à une écoute pure, elle permet le déploiement de menus

\footnotetext{
20 Nous nous inspirons ici de la conférence donnée par Gilles Deleuze en 1987 à Paris, publiée dans Trafic en 1998, dans laquelle le philosophe cherche à définir l'acte de création en répondant à la question : "Qu'est-ce que c'est qu'avoir une idée?».

21 "People think music is what you hear [...]. But music is actually a relationship of values on a page »; notre traduction.
} 
détails aisément percevables par l'auditeur. À l'opposé, les plus larges pinceaux sont les outils adéquats de la composition : "C'est comme une autre façon de peindre, avec un pinceau différent, en exprimant les idées d'une autre manière " (Shore cité dans Willis-Sweete 2011). C'est que Shore doit tenir compte de la forme, de la structure et de la couleur du film. En outre, le type d'écoute exigé par le médium cinématographique diffère de celui adopté dans la salle de concert : une partition trop détaillée, complexe, nuirait sans doute à l'écoute unitaire de l'image.

L'analogie avec la peinture concerne ici le processus créateur. Nous pourrions d'ailleurs la poursuivre non plus en termes d'outils, mais de matériau : point-ligne et mélodie, plan-profondeur et harmonie, valeur plastique (degré d'intensité d'un ton, sa nuance) et intensité du son, couleur et timbre, rythme et durée, espace, densité, texture (Parrat 1993, p. 79, tableau 3). Ne sont-ce pas là autant d'éléments que Shore perçoit dans le film? Du mouvement, des plans, des couleurs, des lumières, le rythme du montage, l'espace du décor, le timbre des voix, la texture des costumes, qui provoquent une musique?

La partition de Crash est un bon exemple pour illustrer ces différents rapports. Le bleu métallique dominant du film inspire ainsi à Shore son instrumentation singulière - « accorder [...] un son à une couleur, le bleu des images de Crash et le son des guitares" (Shore cité dans Joyard et Larcher 2000, p. 35). En outre, la froideur métallique des instruments et du traitement électroniques mêlée à la sonorité chaude des instruments acoustiques (flûte, hautbois, clarinettes, harpes, cordes) fait entendre le rapport entre accident de voiture et sexualité, entre corps métalliques et charnels - l'un des thèmes dominants du film. Ce rapport entre métal et chair est également symbolisé par l'utilisation d'un piano modifié par l'insertion d'objets métalliques entre ses cordes. L'instrument ainsi préparé fait entendre un choc métallique entrant en résonance avec le choc des tôles automobiles du film. De la même manière, l'effet de distorsion parfois appliqué aux guitares peut être perçu comme le reflet sonore du froissement de la tôle, du moins d'une altération de la matière (sonore, organique ou métallique).

Outre des couleurs et des matières, cette partition fait également entendre différents mouvements et ce dès le thème principal qui oppose ainsi trois mouvements sonores, joués simultanément par les trois groupes de guitares/harpe : celui, monolithique, de quintes à vide parallèles (figure 2), auquel s'ajoutent l'ondulation d'un accompagnement continu en arpèges ascendants-descendants - qui résulte de la répétition-variation d'un arpège initial (figure 4) - , puis le mouvement conjoint (ou presque) et sinueux d'une ligne mélodique chromatique tournant autour d'elle-même (figure 5).

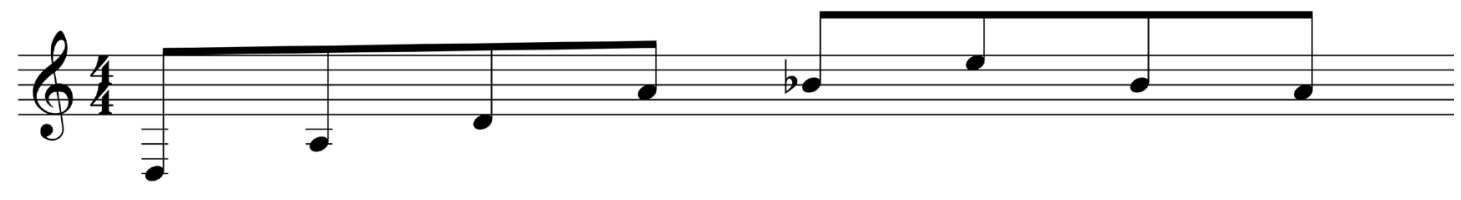

Figure 4: Arpège de Crash - transcription. 
Guitares électriques/harpe $3\left(\downarrow^{\prime}=132\right)$

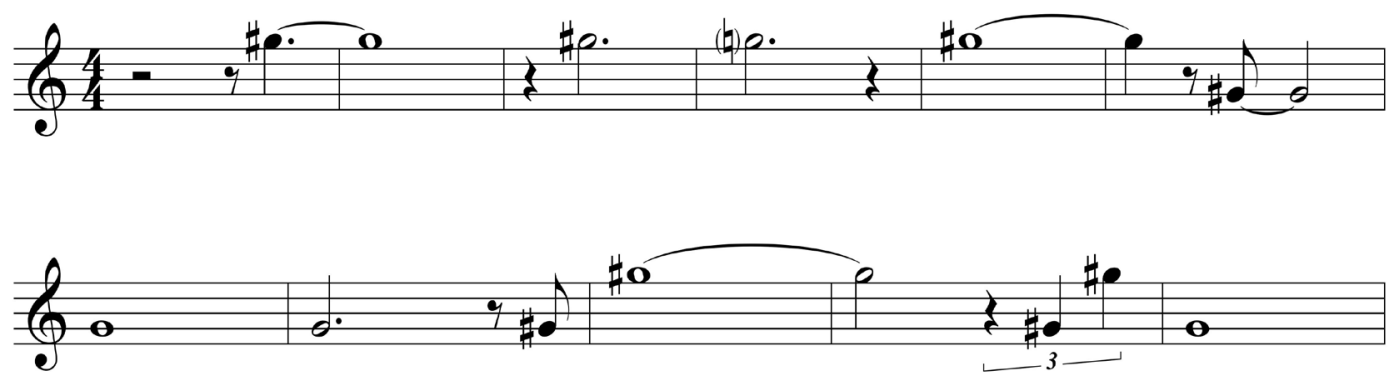

Figure 5 : Ligne mélodique chromatique de Crash (mesures 9 à 19) - transcription.

Tel que nous l'avons démontré ailleurs (Hellégouarch 2012), ces trois éléments (quintes parallèles, arpège, ligne chromatique) vont générer non seulement l'ensemble du thème principal, mais également toute la partition. De plus, l'analyse de celle-ci démontre qu'un seul accord contiendrait en germe l'ensemble de la musique, ses pôles tonaux principaux et ses intervalles fondamentaux, et qu'il pourrait être ainsi perçu comme la traduction musicale même du film : de même que ce dernier se construit à partir d'un accident fondateur, l'ensemble de la partition est générée par un accord originel $^{22}$. Il s'agit en réalité de l'arpège (figure 4), présenté sous sa forme plaquée. Fidèle à sa conception de la musique filmique, Shore a élaboré la musique de Crash en un seul bloc, de manière distancée ${ }^{23}$ et à partir d'un petit nombre d'idée sans cesse développées et amenées toujours un peu plus loin, variées - nous retrouvons donc ici l'influence du jazz et l'idée d'improvisation ${ }^{24}$ qui branche aux impressions laissées par le film des motifs ou phrases musicales qui auront une valeur génétique pour la composition.

L'analogie entre musique et peinture touche enfin le geste, pictural et compositionnel (l'implication du corps) :

Le peintre sent dans ses muscles les mouvements qu'il ferait en suivant avec ses mains ou avec ses yeux la forme de l'objet... Il en est de même des images mentales qui ne sont rien d'autres, selon Sartre, que des impressions de mouvement appréhendées sous forme imagée (Mandelbrojt cité dans Parrat 1993, p. 70).

22 Notons d'ailleurs que cet accord est entendu pour la première fois comme conséquence du tout premier accident du film (l'élément déclencheur), celui de James Ballard et Helen Remington.

23 «Crash a été écrit comme un long morceau que j'ai analysé après l'avoir écrit afin ça fonctionne avec le film. Cette méthode est opposée à celle qui consiste à regarder une scène puis se demander "de quoi cette scène a-t-elle besoin?", puis d'écrire ensuite 40-50 minutes de musique pour organiser le film. » Notre traduction de cet extrait : "Crash was written as a long piece that I analysed after I wrote it so as to make it work in the film. This is opposed to the method of looking at a scene and wondering "what does this scene need?" and then writing 40-50 minutes of music to organize the movie " (Shore cité dans Brophy 1999, p. 4).

24 Dans la partition de Crash, quelques sections sont mêmes destinées à être improvisées par les interprètes. 
Le geste artistique est un geste musculaire : il est pensé à l'avance (face à l'objet), intériorisé (visualisation interne), puis inscrit " avec sa force, sa vitesse, son intensité » (Parrat 1993, p. 70) sur la toile, l'instrument ou le papier. Ce geste musculaire traverse le processus créateur de Shore : c'est la présence du corps (du regard) lors du visionnement du film, la présence de la main sur le clavier et l'enregistreur, lorsqu'il improvise, sur le papier, lorsqu'il compose, sur l'enregistreur multipiste ou l'ordinateur lorsqu'il manipule la matière musicale ainsi obtenue, dans Scanners, Videodrome, Crash ou encore Cosmopolis.

La composition est une action du corps: "L'énergie psychique de l'artiste se transforme directement en énergie musculaire et l'œuvre d'art (arts d'expression) est la trace matérielle esthètisée [sic] du mouvement corporel » (ibid., p. 72). Peindre, déclarait Hans Hartung, en 1954, c'est « agir sur la toile » (ibid.). C'est poser un geste, travailler la matière. Le peintre agit sur la toile, il y imprime son geste, le mouvement, son image mentale : il laisse une trace. De la même manière, Shore extériorise une image mentale, sa visualisation intérieure suscitée par le film, sur l'instrument (premier geste musculaire), le papier (toujours au crayon), l'enregistreur ou l'ordinateur pour modifier la matière sonore. Il agit également directement sur la toile que représente le film en y apposant son geste musical, modifiant ainsi la matière filmique - il fait sonner la couleur, vibrer le mouvement, ajoutant ainsi une autre dimension au film et sa signification - ajouter un autre niveau signifiant.

\section{SCULPTER LE FILM}

Cette notion de l'art comme « concrétisation d'un mouvement corporel » et «joie du geste, de la matière travaillée » (ibid.), nous la retrouvons chez Cronenberg et il n'est pas surprenant qu'il fasse lui-même appel à un autre art pour décrire son processus créateur. Outre l'écriture, il s'agit de la sculpture ${ }^{25}$.

Cronenberg est un inventeur, un sculpteur d'objets en tous genres (souvent érotisés). Dans Dead Ringers, par exemple, il utilise un arsenal d'instruments chirurgicaux inspiré de sculptures en aluminium qu'il avait réalisées au début des années 1970 . Dans le film, Beverly Mantle dessine des instruments destinés à opérer les femmes mutantes et demande à un artiste de les confectionner. Véritables œuvres d'arts, ces instruments sont exposés dans une galerie. De manière générale, à l'intérieur des films, l'art est incarné par différents artistes : le sculpteur Benjamin Pierce (Scanners), l'écrivain Bill Lee (Naked Lunch), la chanteuse d'opéra Song Liling (M. Butterfly) ou encore la créatrice de jeux vidéos Allegra Geller (eXistenZ).

Outre ces personnages, les savants cronenbergiens sont perçus comme des artistes : le savant est un créateur de nouvelles maladies, de nouveaux monstres qui modifient, sculptent les corps des protagonistes. Dans Scanners, par exemple, un médicament développé par le Dr Ruth pour les femmes enceintes confère des pouvoirs télépathiques pouvant agir sur le corps et l'esprit. À travers ses personnages, Cronenberg

25 En 2003, Sébastien Rossignol réalise également un parallèle entre le cinéma cronenbergien et la peinture en comparant l'esthétique du cinéaste à celle de Francis Bacon. 
construit et déconstruit les corps, observe ce qui arrive à la chair martyrisée, arrache la peau pour mieux voir ce qu'elle dissimule. Son cinéma est ainsi aussi visuel que tactile - nous retrouvons cette présence de la main déjà perçue dans le processus créateur de Shore.

Sculpteur de corps, le réalisateur développe un art similaire à celui du body art :

Cronenberg rejoint ici les préoccupations d'un certain art moderne qui fait du corps son médium premier, sa matière première, non commercialisée, totalement libre et affranchie. Déjà, dans les films antérieurs du cinéaste, le gore constituait une variante extrême de l'esthétique du body art, cette forme d'expression où la chair est tout à la fois outil de création et œuvre aboutie. Préoccupé par la reconquête de cet univers de chaos et de contrôle, d'instinct et de raison, il s'en prend film après film à l'un des ultimes tabous de la civilisation contemporaine : le corps (Pompon et Véronneau 2003, p. 178).

De son côté, Sébastien Rossignol établit un parallèle avec l'artiste-performatrice française Orlan qui «utilise son propre corps comme matière première, non pas seulement dans une visée de représentation mais dans une métamorphose » $\left(\right.$ Rossignol 2003) ${ }^{26}$. Mais si Cronenberg est un sculpteur de corps et de matière à l'intérieur du film, il est également un sculpteur de film en tant que tel. Ici, il nous faut revenir à l'improvisation.

Dans le cinéma improvisé, le scénario est conçu comme une matière première ${ }^{27}$, un work in progress, qui contient peu (ou pas) d'indications techniques : "Ces scénarios ouverts sont incompatibles avec le découpage, lieu du programme et de la maîtrise, explique Gilles Mouëllic. Les cinéastes improvisateurs n'y ont donc jamais recours, préférant improviser un découpage in situ» (Mouëllic 2011, p. 23). De la même manière, les scénarios cronenbergiens ne contiennent aucune information technique, le réalisateur préférant produire un texte qui se lit davantage comme une fiction (Breskin 1997, p. 245). On ne retrouve ainsi aucune indication sur l'emplacement ou le mouvement de la caméra :

Eh bien, la raison pour laquelle je n'indique aucune instruction de caméra [dans le scénario] est que je sais que je vais le faire sur la plateau [...]. C'est très palpable et concret pour moi. Ce n'est pas du tout une chose abstraite. Je ne sais pas comment faire un storyboard pour une scène comprenant deux personnes. Je veux impliquer mes acteurs dans la chorégraphie de la scène (Cronenberg cité dans ibid., p. 245-246) ${ }^{28}$.

26 En 2000, Orlan et Cronenberg sont réunis à la galerie Enrico Navarra (Paris) pour une exposition sur « Le corps mutant ». Sur l'utilisation du corps comme matière première, Cronenberg et le body art, voir Baron 2007.

27 Gilles Mouëllic parle de «matière-scénario » (2011, p. 28).

28 "Well, the reason I don't put the camera instructions in there is because I know I'm going to make it up on the set [...]. It's very palpable and tangible to me. It's not an abstract thing at all. I wouldn't know how to make a storyboard of a scene of two people. I want to involve my actors in the choreography of the scene "; notre traduction. 
En effet, Cronenberg ne réalise presque jamais de storyboard - une trace de son autodidactisme. Cette manière de procéder, qu'il qualifie d' "anti-hitchcockienne ", lui confère plus de liberté pendant le tournage (Rodley 1992, p. 153) - c'est le refus de la contrainte autant que de la convention.

Le jour du tournage, Cronenberg n'a donc aucune idée préconçue et reste ouvert à toute possibilité.

Improviser, pour un cinéaste, c'est accepter de ne pas tout savoir avant la prise, c'est faire confiance aux événements imprévus pour atteindre une vérité qui échappe. En comptant sur l'investissement de chaque membre de l'équipe pour inventer avec lui les conditions nécessaires à l'improvisation, le réalisateur refuse d'opposer un savoir qu'il serait seul à posséder à la supposée ignorance de ses collaborateurs réduits au rôle d'exécutants (Mouëllic 2011, p. 33).

L'image surgit ainsi pendant le tournage, "vécu comme expérience » (ibid., p. 28), au contact du matériau et des outils mis à la disposition du cinéaste. Cronenberg ferait ainsi partie de ce que Mouëllic nomme les "cinéastes du tournage ", " qui font confiance au travail d'équipe sur le plateau, acceptant de laisser une grande part à l'improvisation collective » (ibid., p. 17) ${ }^{29}$ - Cronenberg pense la production d'un film comme un travail collectif.

C'est pour décrire ce processus que Cronenberg fait appel à la sculpture :

C'est un processus organique, sculptural, faire un film. On place chaque morceau d'argile, on le lisse, on le palpe, petit à petit. D'où mon rejet $d u$ storyboard. [...] Les images viennent donc plus tard, chez moi. Si ça marche, ça doit venir de l'intérieur. Je suis très inspiré par l'espace, par les lieux, par les visages des acteurs, les costumes qu'on leur fait. Par la lumière que crée mon opérateur. Par tout ça. C'est un processus très plastique, la création des images, au lieu d'être conceptuel (Cronenberg cité dans Labarthe 1999).

Cronenberg puise ainsi son inspiration dans les mêmes éléments cinématographiques que Shore, à la différence que la matière filmique est encore en devenir. Son processus créateur est donc tout à fait similaire à celui du compositeur en termes de visualisation intérieure, improvisation et travail concret de la matière.

Cronenberg s'inspire de la matière dont il dispose pour sculpter, modeler son film : il a donc également besoin d'un ancrage corporel pour créer - écrire le scénario, travailler avec le corps des acteurs, inscrire son propre corps sur le plateau de tournage ${ }^{30}$. À son tour, Shore s'inspire de la matière façonnée par Cronenberg, qui contient déjà

29 L'autre catégorie étant celle des « cinéastes du scénario ", " qui considèrent le tournage comme un moment d'application d'une œuvre dont l'essentiel est déjà contenu dans l'écriture » (Mouëllic 2011, p. 17).

30 «Être un cinéaste improvisateur, c'est mettre son corps dans le flux des événements et tenter de prendre en compte toutes les sollicitations pour jouer, tel un musicien de jazz, la bonne note ou le bon accord au bon moment. Il ne s'agit pas seulement de capter mais aussi de solliciter, d'orienter, de susciter » (ibid., p. 85). 
sa musique, en puissance, afin de lui-même modeler (peindre) la matière musicale. Sa première inspiration constitue la matière sonore de base (les idées issues de l'improvisation) qu'il sculptera à son tour, organisera, plaçant chaque morceau, chaque idée, les reliant, les assemblant pour créer une matière musicale unifiée, dernier morceau d'argile à fondre dans la matière cronenbergienne.

\section{CONCLUSION}

Autodidactes et expérimentateurs, le savoir-faire de Shore et Cronenberg est unique et en constante évolution - comme Shore, le cinéaste est un continuel apprenti qui se renouvelle, expérimente constamment. Il n'en demeure pas moins que ce savoir-faire évolutif contient un certain nombre de constantes : certaines données sont acquises et répétées - une même vision se rejoue de film en film. En outre, les deux collaborateurs comparent respectivement leur travail à celui du sculpteur et du peintre qui travaillent à partir de leurs mains, à même la matière. En ce sens, Shore et Cronenberg peuvent être considérés comme des artisans ${ }^{31}$, mais de leur propre art.

Toutefois, à la différence de l'artisan, Cronenberg et Shore adoptent un processus créateur plastique et organique, l'improvisation, laissant place à la surprise. Pour l'un comme pour l'autre, l'œuvre émerge peu à peu sous leurs yeux (sur l'instrument, le papier, le tournage ou l'écran), tel un produit de leur intuition. En ce sens, ils peuvent être décrits comme des artistes ${ }^{32}$. Pour le cinéaste, ceci est possible dans la mesure où la production filmique est pensée comme un travail collectif ${ }^{33}$ - il peut improviser avec la matière créée par ses collaborateurs à partir de sa vision originelle (le scénario).

Cette étude portait non pas sur les fonctions poétiques, narratives, esthétiques de la musique au cinéma, mais sur le processus de création de films, inséparables de la musique qui les habite. Le discours des artistes a donc été largement pris en considération. Il est cependant nécessaire de garder à l'esprit que leurs témoignages doivent être considérés comme des conjectures, des indicateurs de dimensions à étudier avec attention $^{34}$. Il resterait donc à voir ce que disent également les œuvres.

31 Le processus créateur de l'artisan est mécanique. Pour créer, il dispose d'un plan, d'une idée première qui précède l'œuvre et en règle l'exécution. L'objet final, conforme à sa cause formelle, est reproductible ; il a donc ses limites. Le principal instrument de création de l'artisan est la main. Notons que la figure de l'artisan ne fait pas référence ici à un état professionnel mais à une posture, une façon de penser et pratiquer la création, posture qui peut tout à fait s'inscrire dans un contexte matériel et économique industriel.

32 L'artiste ne limite pas sa création à l'application d'un savoir-faire. Son instrument principal est son esprit, ou plutôt son intuition. Il est le spectateur de son œuvre qui se dévoile peu à peu à lui : "l'idée lui vient à mesure qu'il fait ; il serait même rigoureux de dire que l'idée lui vient ensuite, comme au spectateur, et qu'il est spectateur aussi de son œuvre en train de naître » (Alain 1926, p. 38).

33 «Pour faire du tournage des aventures collectives, les cinéastes improvisateurs s'entourent souvent des mêmes collaborateurs, projet après projet, allant même jusqu'à constituer des troupes fondées sur la complicité et la confiance » (Mouëllic 2011, p. 34).

34 Selon Gilles Deleuze, et tel qu'explicité par Serge Cardinal, les entretiens font partie de l'œuvre d'un auteur. Dès lors, tenter de déterminer l'idée créatrice d'un cinéaste ou d'un compositeur (l'idée problématique qui pousse à la création), c'est considérer leurs créations et ce qu'ils en disent : l'idée est déterminée de film en film, à travers ses répétitions et déterminations, mais aussi d'entretien en entretien. Et tout ce 
Shore est un des rares compositeurs possédant une véritable faculté d'assimilation et une sensibilité à la plastique de l'image rappelant la collaboration d'Eisenstein et Prokofiev. Pour comprendre le processus ici en jeu, l'approche visuelle et plastique du compositeur, il faudrait déterminer l'idée cinématographique équivalente de l'idée musicale, identifier les figures fixées par Shore avant l'improvisation et la composition, décomposer les films en termes de couleur, montage, mouvement, forme, etc. Il s'agirait donc d'essayer de comprendre le passage de l'idée visuelle à l'idée musicale : qu'est-ce qui dans l'image provoque une musique? Pour comprendre un tel phénomène, il serait intéressant de comparer le binôme à des collaborations présentant un processus créateur similaire, notamment en termes d'improvisation. Nous pensons, entre autres, à David Lynch et Angelo Badalamenti (1988-) ou encore Norman McLaren et Maurice Blackburn (1947-1983) ${ }^{35}$.

\section{BIBLIOGRAPHIE}

Adorno, Theodor W., et Hanns Eisler ([1947]1972), Musique de cinéma. Essai, Paris, L'Arche.

Alain (1926), Système des beaux-arts, Paris, Gallimard.

Aufort, Thomas, et Jacky Dupont (2011), «Entretien avec Howard Shore part. 1 », http:/ /lecranmusical. blogspot.ca/2011/07/entretien-avec-howard-shore-part-1.html, consulté le 7 octobre 2014.

Baron, Denis (2007), Corps et artifices. De Cronenberg à Zpira, Paris, L'Harmattan.

Breskin, David (1997), Inner Views. Filmmakers in Conversation, New York, Da Capo Press.

Brophy, Philip (1999), "Howard Shore in Conversation. Composing with a Very Wide Palette ", dans Philip Brophy (dir.), Cinesonic. The World of Sound in Film, North Ryde, AFTRS, p. 1-14.

Brown, Royal S. (1994), Overtones and Undertones. Reading Film Music, Berkeley, University of California Press.

Cardinal, Serge (2010), Deleuze au cinéma. Une introduction à l'empirisme supérieur de l'image-temps, Québec, Presses de l'Université Laval.

Chion, Michel ([2003]2010), Un art sonore, le cinéma. Histoire, esthétique, poétique, Paris, Cahiers du cinéma.

Ciment, Michel, et Hubert Niogret (2007), «Entretien avec David Cronenberg. La réalité, c'est le corps ", Positif, n ${ }^{\circ}$ 561, p. 16-18.

Cook, Nicholas (1998), Analysing Musical Multimedia, Oxford, Clarendon Press.

Deleuze, Gilles (1998), «Qu'est-ce que l'acte de création? », Trafic, n 27, p. 133-142.

Gorbman, Claudia (1987), Unheard Melodies. Narrative Film Music, London, BFI Pub.

Grünberg, Serge ([1992]2002), David Cronenberg, Paris, Cahiers du Cinéma.

Grünberg, Serge (2006), David Cronenberg. Interviews with Serge Grünberg, London, Plexus.

Handling, Piers, et Pierre Véronneau (dir.) ([1983]1990), L'horreur intérieure. Les films de David Cronenberg, Paris/Montréal, Éd. du Cerf/Cinémathèque québécoise.

matériel-là constitue le matériau réflexif du créateur, quel qu'il soit, et participe à la détermination de son idée créatrice, la cohérence de sa pensée et de son œuvre. Voir Cardinal 2010, p. 9-25 et Deleuze 1998.

35 Sur Badalamenti et Lynch, voir Saada 1999. Sur McLaren et Blackburn, voir Hellégouarch 2011b et 2013 . 
Hellégouarch, Solenn (2011a), Entretien avec Howard Shore (1 ${ }^{\text {er }}$ juin).

Hellégouarch, Solenn (2011b) "La pensée de Maurice Blackburn, compositeur de l'ONF », www.creationsonore.ca/wp-content/uploads/2014/10/travaux_solenn-hellegouarch_mauriceblackburn-compositeur-de-lonf.pdf, consulté le 7 octobre 2014.

Hellégouarch, Solenn (2012), " Pour une relecture de Crash (1996), de David Cronenberg. La partition composée par Howard Shore », www.creationsonore.ca/creation-sonore.php?articleset-communications, consulté le 7 octobre 2014.

Hellégouarch, Solenn (2013), «A Phanthasy (1948-1952), de Norman McLaren. Quatuor pour son synthétique et saxophones, de Maurice Blackburn ", www.creationsonore.ca/wp-content/ uploads/2014/10/travaux_solenn-hellegouarch_a-phantasy-de-norman-mclaren.pdf, consulté le 7 octobre 2014 .

Joyard, Olivier, et Jérôme Larcher (2000), «Shore-Cronenberg. L'état de grâce », Cahiers du cinéma $\mathrm{n}^{\circ} 551$, p. 34-35.

Kalinak, Kathryn (1992), Settling the Score. Music and the Classical Hollywood Film, Madison, University of Wisconsin Press.

Kassabian, Anahid (2001), Hearing Film. Tracking Identifications in Contemporary Hollywood Film Music, New York, Routledge.

Labarthe, André S. (1999), "David Cronenberg. I have to Make the World Be Flesh ", http://worldismine.free.fr/articles/david_cronenberg/index.htm, consulté le 7 octobre 2014.

Lauliac, Christian (2002), "Howard Shore. La lumière passe de l'œil au cerveau », Positif, n 502, p. 93-96.

Lissa, Zofia (1965), Ästhetik der Filmmusik, Berlin, Henschelverlag.

Mouëllic, Gilles (2011), Improviser le cinéma, Crisnée, Yellow Now.

Parrat, Jacques (1993), Des relations entre la peinture et la musique dans l'art contemporain, Nice, Z'éditions.

Pompon, Géraldine, et Pierre Véronneau (2003), David Cronenberg. La beauté du chaos, Paris, Éd. du Cerf.

Reynolds, Cory (2001), " Howard Shore », www.indexmagazine.com/interviews/ howard_shore.shtml, consulté le 7 octobre 2014.

Rodley, Chris (1992), Cronenberg on Cronenberg, Toronto, Alfred A. Knopf Canada.

Rossignol, Sébastien (2003), " Le cinéma de David Cronenberg et la peinture de Francis Bacon. Regards croisés ", Mémoire de maîtrise, Université de Toulouse II, http://baconcronenberg.free.fr/, consulté le $1^{\text {er }}$ octobre 2014.

Saada, Nicolas (1999), « "Tout est affaire de mélodie”. Entretien avec Angelo Badalamenti », Cahiers du cinéma, $\mathrm{n}^{\circ}$ 540, p. 60-63, www.davidlynch.de/cahlent3.html, consulté le 20 janvier 2015.

Schelle, Michael (1999), The Score. Interviews with Film Composers, Los Angeles, Silman-James Press.

Small, Mark (1999), « Howard Shore. Subliminal Scores », dans Mark Small et Andrew Taylor, Masters of Music. Conversations with Berklee Greats, Boston, Berklee Press, p. 258-267.

Temmerman, Jan (2003), «Howard Shore ", dans Ray Bennet et al., Moving Music. Conversations with Renowned Film Composers, Tielt, Lannoo, p. 92-99.

Théberge, Paul (2004), " "These are my Nightmares", Music and Sound in the Films of David Cronenberg ", dans Philip Hayward (dir.), Off the Planet. Music, Sound and Science-fiction Cinema, Eastleigh, John Libbey, p. 129-148.

Willis-Sweete, Barbara (2011), A composer's Dream, www.onf.ca/film/reve_d_un_compositeur, consulté le 7 octobre 2014. 